\title{
Real-Time Detection of Unstable Control Loop Behavior in a Feedback Active Noise Cancellation System for In-Ear Headphones
}

\author{
Sven Höber, Christian Pape, Eduard Reithmeier \\ Institute of Measurement and Automatic Control, Leibniz University Hanover, Hanover, Germany \\ Email: sven.hoeber@imr.uni-hannover.de
}

Received 10 November 2015; accepted 14 December 2015; published 17 December 2015

Copyright (C) 2015 by authors and Scientific Research Publishing Inc.

This work is licensed under the Creative Commons Attribution International License (CC BY). http://creativecommons.org/licenses/by/4.0/

(c) (i) Open Access

\begin{abstract}
Active noise controls are used in a wide field of applications to cancel out unwanted surrounding noise. Control systems based on the feedback structure however have the disadvantage that they may become unstable during run-time due to changes in the control path-in this context including the listener's ear. Especially when applied to active noise cancellation (ANC) headphones, the risk of instability is associated with the risk of harmful influence on the listener's ear, which is exposed to the speaker in striking distance. This paper discusses several methods to enable the analysis of a feedback ANC system during run-time to immediately detect instability. Finally, a solution is proposed, which identifies the open loop behavior parametrically by means of an adaptive filter to subsequently evaluate the coefficients regarding stability.
\end{abstract}

\section{Keywords}

Active Noise Control, Feedback, Stability, Real-Time Analysis

\section{Introduction}

Noise can interfere, adversely affect or even be harmful. In this context, the use of hearing protection or headphones with active noise cancellation (ANC) derives a substantial gain in comfort. Besides pilot headsets, this technology is also increasingly being offered in commercially available headphones to reduce noise especially when traveling or at work. While the current selection of ANC headphones is mainly limited to circumaural models, the concept has been extended to the more compact in-ear headphones in recent years. For the technical implementation of ANC systems based on the feedback structure, the interference signal of noise and speaker sound is measured by an internal microphone and fed back to a controller. The problem with the feedback 
structure is the risk of instability, which is expressed in an overdrive and eventual destruction of the speakers or even damage of the user's inner ear. In [1] for example, this problem is overcome by limiting the controller gain. Additionally, practical applications in general limit the actual speaker output or at least deactivate the controller output in case of an overdrive. Thus, conventional solutions either act preemptively or in response to the already unstable state, still exposing the listener to a potential danger. This results in the need to continuously observe the behavior of the control loop during run-time, in order to detect the possibly occurring unstable state at an early stage. In this paper, various methods are compared, which yield an estimation of the control loop state on the basis of the microphone and speaker signals to realize a real-time analysis.

\section{System Setup}

In the following, a feedback control system for active noise cancellation in in-ear headphones is investigated. A schematic setup of the system is shown in Figure 1. The purpose of the system is to minimize the error $e(n)$, which results from the interference of the speaker sound $u(n)$ and the disturbing background noise $d(n)$ at the error microphone. For this, the speaker is driven by the controller $C(z)$ with the actuating variable $y(n)$; the entire control is implemented on a digital signal processor (DSP). The acoustical domain (within the dashed line box) is included in the control path $S(z)$, in ANC applications referred to as secondary path [2]. The equivalent block diagram of the described structure is shown on the right in Figure 1.

The secondary path, however, continuously changes even by slight variations of the headphones' position in the ears, possibly causing the control loop to become unstable [3]. The next sections deal with the design of methods, which detect the characteristic system behavior due to those critical changes by evaluating the measurable signals.

\section{Analysis of the Control Loop}

\subsection{Frequency Domain Based Methods}

The analysis of the system in the frequency domain initially requires a Fourier transform of the signals. To perform this during run time, the short-time FFT is used [4]. Subsequently the ratio of the transformed signals yields the corresponding transfer behavior. The behavior of the closed system can then be derived from the open loop transfer function

$$
G_{\mathrm{o}}\left(e^{j \omega}\right)=S\left(e^{j \omega}\right) C\left(e^{j \omega}\right)=\frac{U\left(e^{j \omega}\right)}{E\left(e^{j \omega}\right)} .
$$

This transfer function can, for example, be displayed in a Nyquist plot and subsequently be evaluated geometrically. The stability of the system is then estimated by applying the simplified Nyquist criterion, according to which the locus of a stable system must intersect the real axis to the right of the critical point $(-1+j 0)$ [5]. Thus, at this intersection with $\omega_{180}=\left.\omega\right|_{\operatorname{Im}\left\{G_{0}\right\}=0}$ we obtain the condition

$$
\operatorname{Re}\left\{G_{0}\left(e^{j \omega_{180}}\right)\right\}>-1
$$

Applied to the investigated system, first of all the signals of the speaker $u(n)$ and the error microphone $e(n)$ are continuously transformed into the frequency domain to estimate the transfer function according to Equation (1). Then the real and the imaginary part of the transfer function are iteratively analyzed regarding Equation (2) to check whether the control loop becomes unstable.
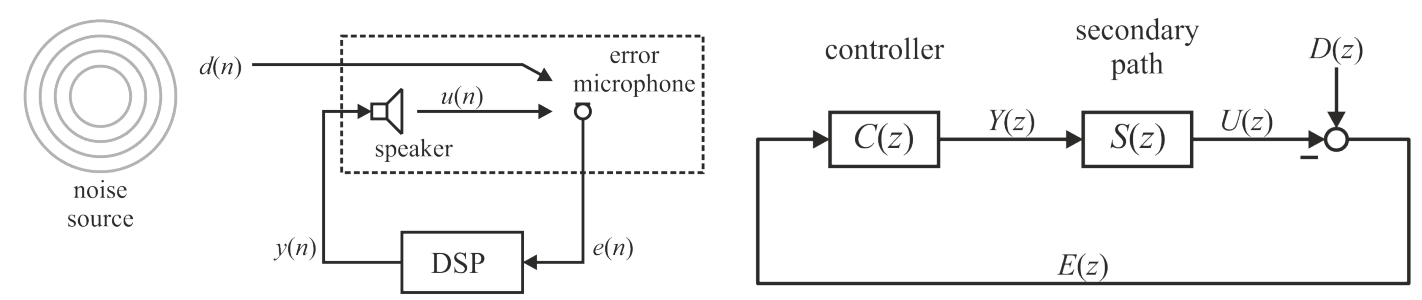

Figure 1. Schematic of the feedback ANC system (left) and block diagram of the equivalent control loop (right). 
When using the Nyquist stability criterion, the system can alternatively be analyzed in a Bode diagram. The estimation of its behavior is then based on the amplitude and phase component of the transfer function. Still, the critical point is crucial, which results in the constraint for stability

$$
\varphi\left(\omega_{D}\right)=\arg \left\{G_{\mathrm{o}}\left(e^{j \omega_{\mathrm{D}}}\right)\right\}>-180^{\circ} .
$$

At the gain crossover frequency $\omega_{D}$, the magnitude crosses the value of $0 \mathrm{~dB}$. To check whether the system is unstable (that is, the constraint (3) is satisfied or not) the phase $\varphi(\omega)$ of the transfer function is now continuously evaluated.

\subsection{Time Domain Based Methods}

Since the proposed methods in the frequency domain inherently cause a high computational effort due to the FFT, alternative methods operating in the time domain (which hence do not require the transfer function of the open loop) are investigated in the following. Now, the power $P_{y}(n)$ of the actuating variable signal $y(n)$ is evaluated, which merely requires an estimation of the power in place of a transformation during each sample. The principle of detecting instability is now based on the characteristics of unstable systems to cause the transferred signals to rise unboundedly, resulting in an immediate and monotonic increase of the signal power [6]. This effect is used in the following methods as an indicator for unstable behavior. However, since the signal power may also increase by the dynamics of the disturbance, first of all a parameter must be defined, which provides information about the cause of the increase. From this, the condition

$$
\frac{\mathrm{d}}{\mathrm{d} t} P_{y}(n)>\delta>0
$$

can be derived, according to which the control loop is presumed unstable if the power changes by more than a positive value $\delta$.

Since this threshold is difficult to quantify, in the first approach an adaptive tolerance band is defined instead, which adapts to the current power level with a selectable fixed delay $k$. When the control loop becomes unstable, the power is expected to increase rapidly enough to cross the upper boundary of the tolerance band. The boundaries are estimated from prior samples of the power

$$
B_{ \pm}(n)=\left(1 \pm \Delta_{P}\right) \cdot P_{y}(n-k)
$$

with the tolerable difference - the width of the band- $\Delta_{P}$.

Instead of evaluating the degree of the increase-determined by $\delta$ - an alternative approach is to quantify the total amount of increases in a fixed time interval. This is actualized by counting the positive and negative changes in slope per interval and checking, whether within the respective interval a certain amount of positive changes is exceeded. For ease of application, the estimated power is first discretized logarithmically; that is, the range of discrete power values is equally spaced per decade. Then two counters detect the rising and falling edges $N_{\mathrm{e}^{+}}$and $N_{\mathrm{e}-}$ of the discretized power. If either a certain amount of falling edges is reached or the time interval (determined by a third, free-running counter with the cycle time $t_{C}$ ) has passed, both edge counters are reset to zero. If, on the other hand, a certain amount of rising edges is reached within the interval, the control loop is presumed unstable and the estimated state is displayed respectively.

\subsection{Combined Methods}

As mentioned in the previous discussions, a real-time implementation of the system analysis in the frequency domain is problematic because of the required FFT. On the other hand, the methods evaluating the signal power in the time domain substantially depend on the parameter sets and are not necessarily correlated with the control loop behavior. Consequently, a method which permits detection of unstable behavior in the time domain based on the transfer function of the open loop is desirable.

In the following approach, the open loop is identified parametrically using an adaptive FIR filter $\hat{G}_{0}(z)$. By applying the determinant criterion of Jury, the coefficients of the filter are then evaluated to check the stability of the system. The corresponding algorithm is described in detail in [6]. Like the Hurwitz criterion for continuous time systems, the Jury stability test evaluates the characteristic polynomial of the digital system 


$$
1+\hat{G}_{0}(z)=1+a_{m} z^{m}+\cdots+a_{1} z+a_{0}
$$

which, in the case of an FIR filter, merely consists of the identified filter's coefficients $a_{i}$. It is continuously adapted by using the LMS algorithm, which can be realized with low effort in implementation and computing power [2] [7]. The coefficients are then evaluated by the algorithm to check the system state.

\section{Simulation Results}

In this section, the proposed methods are tested in simulations. To induce the unstable state in the simulation model, a delay time is added to the secondary path after half the simulation time $(t=0.5 \mathrm{~s})$. The methods are now supposed to detect the state change at this time.

First, the "Nyquist method" is tested. For this, the required signals $u(n)$ and $e(n)$ are continuously fed to the respective algorithm described in Section 3.1 while observing the estimated state (Figure 2). As intended, the method signalizes that the system is unstable for $t>0.5 \mathrm{~s}$. The two peaks apart $(t<0.5 \mathrm{~s})$, which do not reflect the actual system state, result from phase errors. The corresponding variations of the phase especially cause the alternative "Bode method" to yield false results (Figure 2). It too is based on the nyquist criterion, however, as shown in the resultant plot, the stable control loop is often estimated as unstable and vice versa-what is even more serious. That means the instability is not detected continuously which makes the Bode method not feasible.

Now, the power estimation based methods are investigated. Figure 3 shows the simulation results of the estimated power $P_{y}(n)$ and the upper boundary $B_{+}(n)$ of the tolerance band derived from Equation (5). The
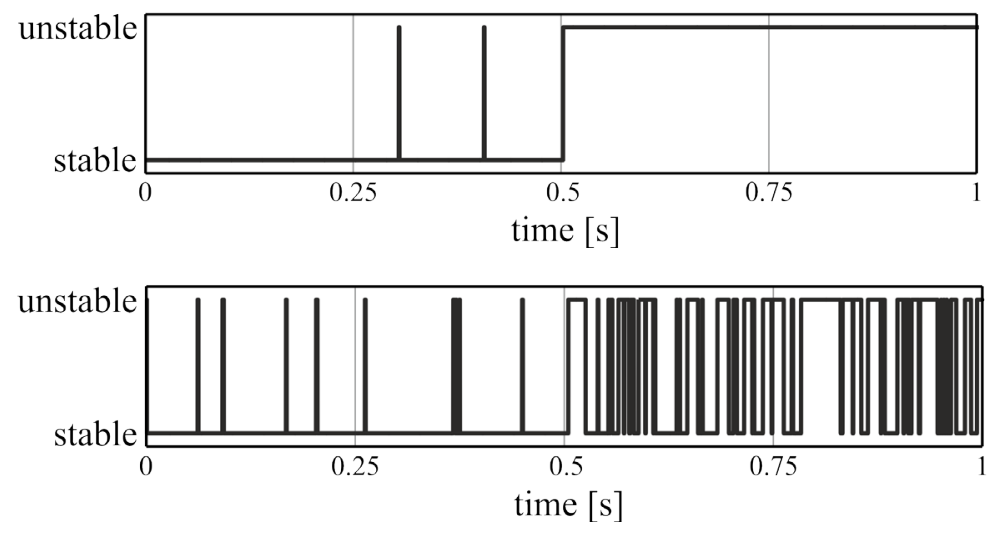

Figure 2. Estimated control loop state using the Nyquist method (top) and the Bode method (bottom).
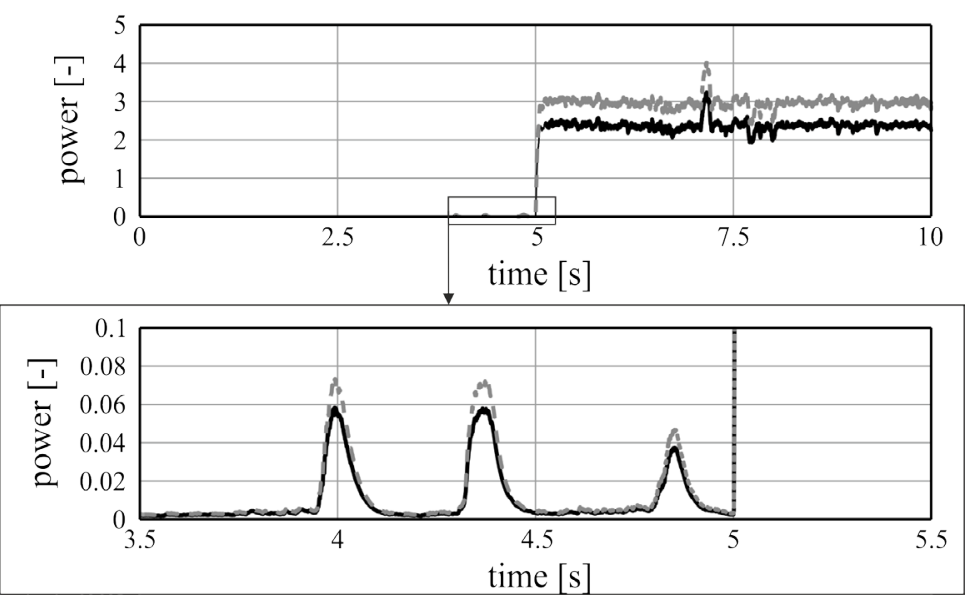

Figure 3. Estimated power of the actuating variable signal (black) and resultant upper boundary (gray). 
enlarged detail reveals how the adaption of the band ensures that irrelevant increases of the power (which are not correlated with the system behavior) do not cross the boundaries. The increase caused by the instability ( $t \geq$ $0.5 \mathrm{~s}$ ) on the other hand, results in a peak of the estimated state (Figure 5) - the simulation time is now $10 \mathrm{~s}$, as needed to depict the power rises adequately. The state immediately falls back to "stable" though because the power reaches its maximum given by the limiting of the actuating variable, eventually causing the power to remain within the tolerance band. However, this result shows that the tolerance band method generally enables the detection of the unstable behavior. The disadvantage of this method is its dependency on the adjustment of the parameters $k$ and $\Delta_{P}$-an optimal parameter set is difficult to find.

Figure 4 and Figure 5 present the results obtained with the alternative edge counter approach. The simulation shows that also with this method, the instability of the control loop is detected immediately. Like the previous method, however, its functionality is highly dependent on the parameters (cycle time $t_{C}$ and threshold values for $N_{\mathrm{e}+}$ and $N_{\mathrm{e}-}$ ) either.

The previous simulations have shown that the approaches in the frequency and time domain both generally enable the estimation of the system state and thus the detection of instability. Their inherent disadvantages (required FFT and dependency on parameters), which make an implementation difficult, are supposed to be omitted by the combined method using the parametrical identification of the open loop. However, simulations have shown that the frequency response of the model filter does not approximate the one of the real open loop sufficiently. This problem has been attributed to the properties of the disturbance $d(n)$. In the case of stationary white noise, for example, the achieved approximation is much better than with the (colored and transient) ambient noise used in all simulations. These parameters affect the adaptation process of the filter significantly. Since the ambient noise reflects more realistically conditions though, it is still used in the following simulations.
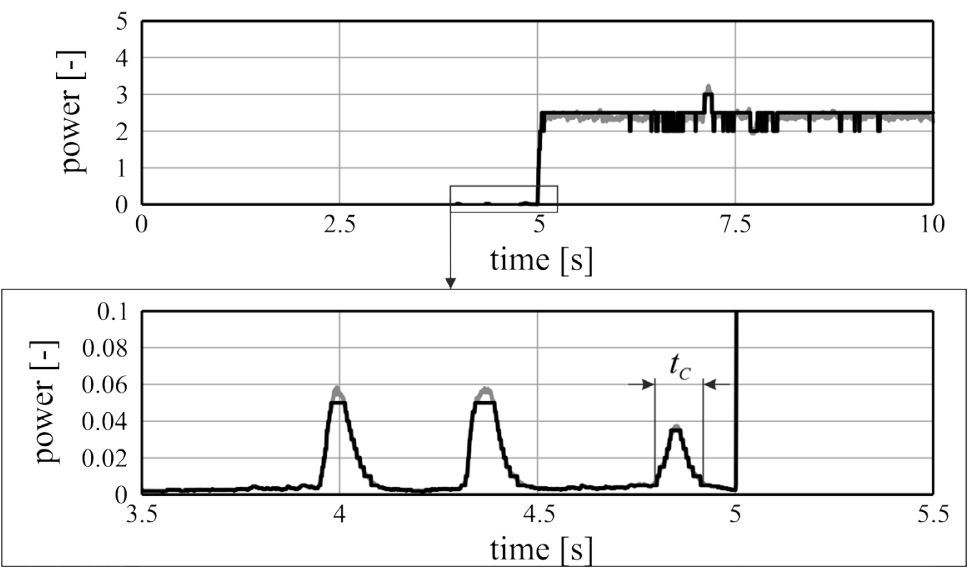

Figure 4. Estimated power of the actuating variable signal (gray) and resultant discretization (black).
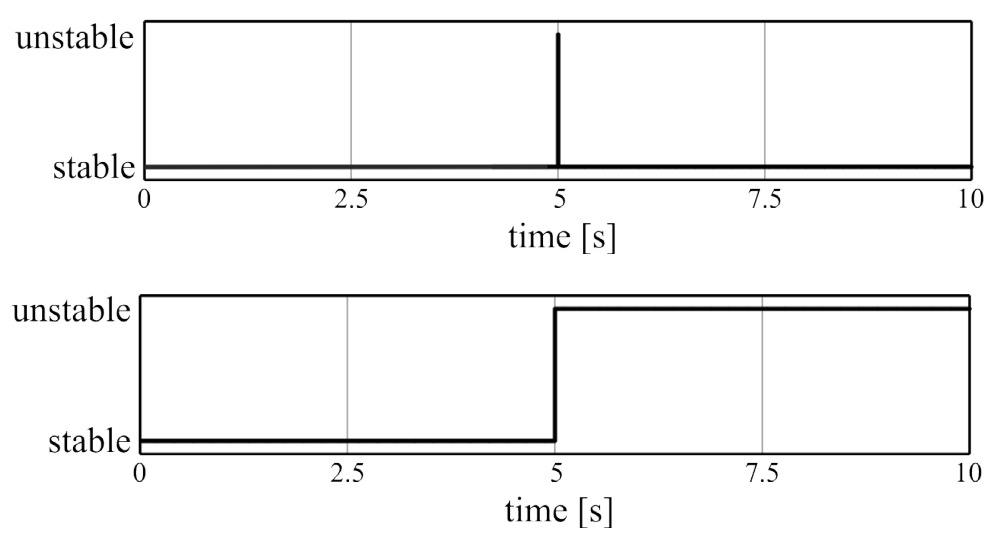

Figure 5. Estimated control loop state using the tolerance band method (top) and the edge counter method (bottom). 
Because of the previously mentioned issues with the open loop identification, the Jury stability test also yields no feasible results at first glance. On closer inspection, however, there is a change in the run-time of the algorithm depending on the system state. More precisely, the index-meaning the value of the loop variable within the algorithm - , at which the iterative estimation of the determinants (see [8]) stops, changes when the control loop becomes unstable. This is shown in Figure 6. Furthermore, the instability of the control loop also becomes apparent by observing the filter coefficients of the model filter directly. It has shown that the coefficients distribution approximately has the shape of a harmonic oscillation (Figure 7). If the plot is interpreted as an oscillation diagram, the state of the control loop can directly be derived from the zero crossings. As shown in Figure 8, the amount of zero crossings increases after the destabilization - that is, for $t>5 \mathrm{~s}$. By defining a threshold for the zero crossings of the coefficients distribution or the "stop index" of the Jury algorithm, these two effects can be used for a robust method to check the stability of the control loop at run-time.

\section{Conclusions}

This paper proposes methods to evaluate the signals of a control loop for ANC in in-ear headphones to detect an occurring instability of the system at run-time. It was shown that the analysis in the frequency domain —on the basis of the open loop behavior-generally allows the appliance of conventional methods such as the Nyquist criterion. However, regarding the sampling frequency of the DSP $\left(f_{S}=40 \mathrm{kHz}\right)$, the high computational effort of the necessary FFT makes a real-time implementation not feasible.

On the other hand, the methods in the time domain—on the basis of signal power estimation-require significantly lower effort in computation and implementation. Yet, their functionality and hence their robustness substantially depend on the parameter sets. Furthermore, the effect of the rapid increase in signal power used for instability detection is not necessarily correlated with the control loop behavior.

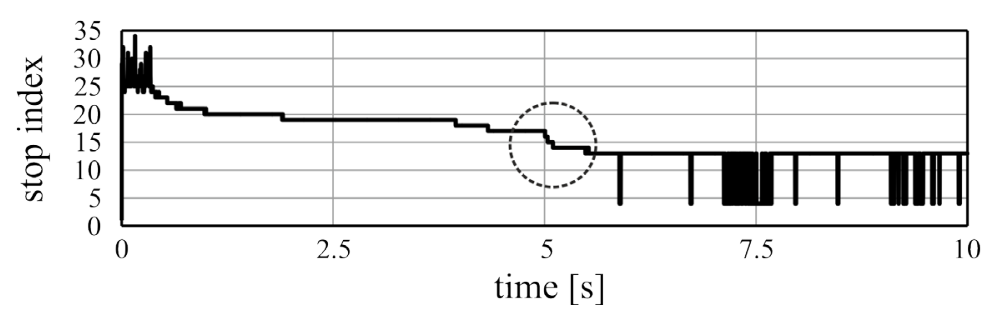

Figure 6. "Stop index" of the Jury stability test algorithm over simulation time.
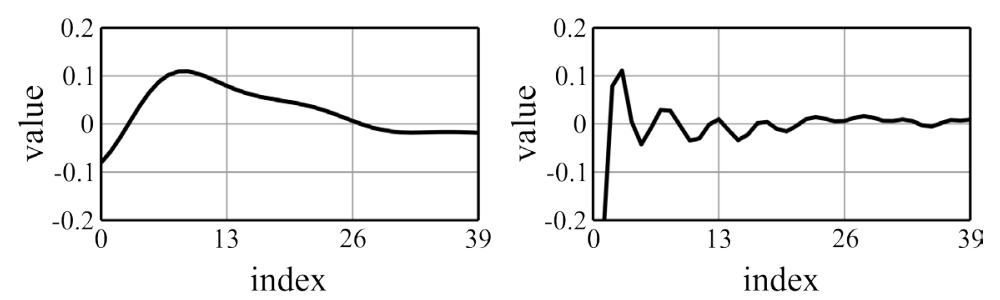

Figure 7. Coefficients (linearly interpolated) of the model filter with stable (left) and unstable loop (right).

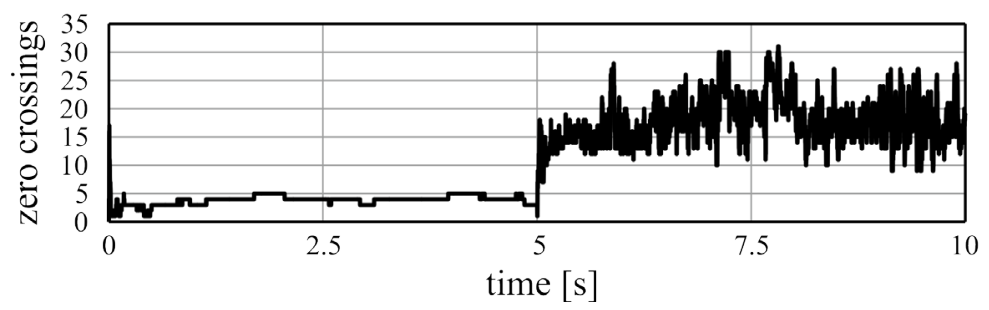

Figure 8. Amount of zero crossings of the coefficients distribution dependant on the control loop state. 
Finally, the combination of the frequency- and time domain-based methods led to a solution, which evaluates the coefficients of an adaptive filter approximating the open loop behavior parametrically. To subsequently check the stability of the model filter, the determinant criterion of Jury was used. Here, however, there were difficulties because the convergence of the adaptive filter and thus the identification of the open loop were impaired by the highly transient properties of the disturbance. Nevertheless, the detection of instability by means of the Jury test was generally possible. Furthermore, it was shown that the state of the control loop can alternatively be estimated by simply observing the distribution of the coefficients of the model filter.

\section{Future Work}

Firstly, the two combined approaches are to be implemented and evaluated experimentally with the real ANC system to substantiate the presented simulation results. However, since the principle of the filter based identification and evaluation of the open loop is suitable for all linear systems, the proposed solution can potentially be utilized for other control applications also.

Furthermore, if the "unstable" state is detected, the system has to be stabilized subsequently. This problem could be solved by observing and controlling the gain of the actuating variable-yet resulting in a decrease of the performance of the control system. An alternative approach would be to compensate for the change in the control path-meaning the distortion of the frequency response-which has caused the closed loop to become unstable originally. This issue is to be researched in detail in future work, too.

\section{References}

[1] Bruhnken, C. (2015) Adaptive Feedback-Regelung zur aktiven Lärmreduktion mit In-Ear-Kopfhörern. PZH Verlag, Garbsen.

[2] Kuo, S.M. and Morgan, D.R. (1996) Active Noise Control Systems: Algorithms and DSP Implementations. John Wiley \& Sons, Inc., New York.

[3] Bruhnken, C., Priese, S., Foudhaili, H., Peissig, J. and Reithmeier, E. (2012) Design of a Feedback Controller for Active Noise Control with In-Ear Headphones. Proceedings of Meetings on Acoustics, 18, Article ID: 040003. http://dx.doi.org/10.1121/1.4755455

[4] Meyer, M. (2003) Signalverarbeitung. Friedr. Vieweg \& Sohn Verlag/GWV Fachverlage GmbH, Wiesbaden.

[5] Lunze, J. (2014) Regelungstechnik 1. Springer, Berlin and Heidelberg. http://dx.doi.org/10.1007/978-3-642-53909-1

[6] Fliege, N. (1991) Systemtheorie. Teubner, Stuttgart. http://dx.doi.org/10.1007/978-3-663-05933-2

[7] Kammeyer, K.-D. and Kroschel, K. (2012) Digitale Signalverarbeitung. Vieweg \& Teubner, Wiesbaden. http://dx.doi.org/10.1007/978-3-8348-8627-9

[8] Lutz, H. and Wendt, W. (2012) Taschenbuch der Regelungstechnik. Harri Deutsch, Frankfurt am Main. 